\title{
Uso del desincrustante magnético (DM) para mejorar la calidad del agua en la industria
}

\author{
Carlos Gálvez Vidaurre \\ Skynet Environmental Perú \\ Ingeniería Industrial n. 28, 2010, ISSN 1025-9929, pp 139-154 \\ Recibido: 15 de junio del 2010 / Aprobado: 19 de julio del 2010
}

RESUMEN: Con el uso del desincrustante magnético (DM) se pueden reducir las incrustaciones calcáreas o deposiciones que se forman en cualquier tubería, maquinaria o equipo industrial donde se presenten los problemas de agua dura, alargando la vida útil de dichos implementos y mejorando la solubilidad de distintas sales, principalmente de carbonato cálcico. Por otro lado, con el uso del DM se han observado variaciones en los valores de $\mathrm{pH}$, de la conductividad eléctrica y tensión superficial del agua, haciéndola "más húmeda", por lo que se ahorraría significativamente el uso de productos químicos para tratar el agua.

Palabras clave: calidad del agua / tratamiento magnético / polarización / desincrustante / ambiente

\section{Use of magnetic descaler (MD) for industrial water quality improvement}

ABSTRACT: The use of the magnetic descaler (MD) can reduce the calcareous depositions that are formed in any pipeline, machinery or industrial equipment where the problems of hard water are presented, extending the useful life of the pipe lines and improving the water properties as solubility, mainly calcium carbonate. On the other hand, with the use of the MD variations have been observed in the values of $\mathrm{pH}$, the electrical conductivity and surface tension of the water, making it "wetter water" and significantly can reduce the use of water treatment chemicals.

Key words: water quality / magnetic treatment / polarization / descaler / environment 


\section{ANTECEDENTES}

Si bien las primeras observaciones del efecto de la magnetita en la reducción de sarro calcáreo datan de principios del siglo XIX, el tratamiento magnético del agua para disminuir los depósitos calcáreos en calderas y mejorar sus propiedades físicas, entre ellas el sabor, así como la reducción de la tensión superficial, ha sido utilizado en Europa y especialmente en Rusia en los últimos setenta años. La tecnología magnética creció en esa área debido a la poca disponibilidad y altos costos de los productos químicos. Paralelamente se observó un mayor interés científico por desarrollar nuevas tecnologías para el tratamiento del agua dura. En Estados Unidos siempre se han usado "respuestas químicas" a los problemas del agua debido a que dicho país cuenta con vastas reservas, grandes volúmenes de procesamiento y distribución de productos químicos, así como de una tradicional industria química rentable. Sin embargo, con los altos costos de estos productos y los aun más altos costos por daños ocasionados al ambiente, los productos químicos no ofrecen en la actualidad una solución plausible para resolver nuestros problemas cotidianos.

Aunque el primer dispositivo para el control de incrustaciones data de 1890 —según la patente $\mathrm{N}^{\circ} 438.579$ de los inventores Albert B. Frunce y Samuel G. Cabell- usando medios eléctricos, el tratamiento magnético de líquidos es reconocido formalmente desde mediados del siglo pasado, con el trabajo realizado por el inventor belga Theofile Isidore Sophie Vermeiren, originario de Deurne-Anvers, Bélgica, el cual registró su solicitud sobre el "Dispositivo para tratamiento magnético de líquidos" el 24 de diciembre de 1948, otorgándose la patente en la oficina de patentes de Estados Unidos el 22 de setiembre de 1953. Sin embargo, el trabajo previo de Vermeiren fue el desarrollo de un aparato eléctrico para prevenir la formación de incrustaciones calcáreas por efecto del agua dura, el cual fue registrado en Bélgica con el número de serie 700.595, el 6 de octubre de 1945. En este trabajo se explica la influencia de un campo electromagnético para tratar agua y las líneas de fuerza o densidad de flujo magnético generado al energizar un solenoide, que era alimentado por una fuente de corriente alterna, según se describe en la patente de Estados Unidos $\mathrm{N}^{\circ}$ 2.596.743, otorgada el 13 de mayo de 1952 .

Posteriormente se llevaron a cabo diversos experimentos, como el de la firma United States Testing Company, Inc., que realizó una 
prueba para determinar la efectividad del tratamiento magnético del agua en la prevención de incrustaciones calcáreas en calderos. Luego del análisis de las sales residuales en cada caso, se encontró una diferencia distintiva. El análisis de difracción de rayos $\mathrm{X}$ en los residuos de agua no tratada magnéticamente mostró que las especies de cristal dominante eran de tipo de sulfato de calcio y silicato de calcio, mientras que en los residuos de agua tratada magnéticamente las especies de cristal dominante fueron de tipo carbonato de calcio y sulfato de calcio, que son elementos no corrosivos. Por esa época también fueron publicados diversos estudios realizados en Rusia, donde se afirmaba que el tratamiento magnético de agua era usado extensivamente y con tremendos efectos económicos. Asimismo, el fenómeno de tratamiento magnético del agua ha sido científicamente investigado en Gran Bretaña, donde los dispositivos instalados dieron los mismos resultados favorables que en el resto de Europa.

Por otro lado, la ARPA (Advanced Research Projects Agency) del Departamento de Defensa de Estados Unidos dio como resultado de sus investigaciones que el tratamiento magnético del agua reduce la cinética del proceso de cristalización. Dicho en términos sencillos, significa que elimina la calcificación y los problemas asociados con el agua dura. Otros efectos observados son la reducción de depósitos de sal, intensificación del proceso de coagulación y cristalización, mejora de la función bactericida de los desinfectantes, aumento de la eficiencia de las resinas de intercambio iónico, aceleración en la solidificación de cementos, incremento de densidad y dureza de piezas fundidas, etcétera. Adicionalmente, cuando cualquier superficie de transferencia de calor se encalicha ${ }^{1}$ (formación de sarro) se produce un aislamiento calcáreo que reduce la eficiencia, aumenta el consumo de combustible (mayores emisiones de $\mathrm{CO}$ ) e incrementa los costos de mantenimiento de la maquinaria y del equipo. Por lo tanto, es importante realizar un trabajo efectivo al tratar el agua, no solo por sus implicancias económicas sino para reducir al mínimo el impacto de las emisiones gaseosas en el ambiente.

El tratamiento magnético del agua - un método no químico para tratar aguas duras- es usado extensivamente en todo el mundo con

1 Caliche o sarro es el nombre de las incrustaciones de cristales de calcita en cualquier superficie donde circule agua fría o caliente. 
importantísimos efectos económicos. Otro estudio llevado a cabo por la NASA comparó el agua tratada magnéticamente contra la corrosión. Los resultados fueron excelentes: usando los productos químicos, el índice de corrosión fue de una a cincuenta mils por año, cuando se consideraba aceptable un índice de cuatro mils por año. El resultado con tratamiento magnético fue de un índice de 0,0 mils de corrosión por año. ${ }^{2}$

De acuerdo con el artículo aparecido en la edición de abril de 1990 del Plumbing Engineer: "Tratamiento de agua-La Guía del Ingeniero", para tratar 1.000 galones de agua con dureza de 20 grains $^{3}$ se requieren 20 libras de químicos, usualmente sales. El problema generado por este método es que cada vez que se utilicen sistemas ablandadores de agua que usen sal, serán descargadas toneladas de salmuera en las redes de drenaje (desagües), contaminando los acuíferos locales (filtración) para siempre y deteriorando los equipos de purificación de agua. El uso diario de químicos para ablandar el agua llevará a que las capas de sales del subsuelo continúen creciendo.

En la década de 1990, el Estado de California, Estados Unidos, líder en asuntos ambientales, restringió el uso de ablandadores de agua basados en sal en 28 municipios. En dichas zonas está estrictamente prohibida la descarga de residuos de salmuera en el sistema de desagües públicos. El resultado es beneficioso: se ahorra agua usada para regenerar el ablandador de agua. El agua no usada puede destinarse al consumo humano, a la actividad agrícola o con otros fines más beneficiosos.

El tratamiento magnético del agua en la industria tiene como efecto inmediato el ahorro de energía, ya que el sarro o caliche es un aislante térmico que reduce la transferencia de calor desde los tubos de una caldera hacia el agua. Por ejemplo: un grosor de incrustaciones de $1 / 10$ de pulgada reduce la transmisión de calor en $38 \%$. Asimismo, un grosor de incrustaciones de $1 / 2$ pulgada causa un $60 \%$ de pérdida en eficiencia en un intercambiador de calor. Por otro lado, el paso de agua a través de un campo magnético focal balancea el PH hacia 7,0 ya que se observó este efecto en un test conducido por Crippen Laboratories, Inc., en 14 pozos de la Municipalidad de Wilmington, Delaware, Estados Unidos. El efecto amortiguante fue evidente en 12 de las 14 muestras de agua de pozo tratadas magnéticamente, donde se empleó un

$21 \mathrm{mil}=25 \mu \mathrm{m}$. 
magneto de bajo poder. De igual modo, en la edición de la revista Science News del 6 de setiembre de 1980, publicada por la Science Service, Washington, D.C., Frank Ellingson, un profesor del staff de Ingeniería Química de la Universidad John Hopkins de Baltimore, afirma que "En algunos casos, la cantidad de incrustaciones formadas fue reducida por un factor mayor a 100".

Amplia documentación del efecto de agua tratada magnéticamente se detalla en el reporte AD-757-887, Magnetic Treatment of Water, distribuido por el National Technical Information Service del Department of Commerce, Springfield, Virginia, Estados Unidos. Otro reporte es el editado por los doctores Donaldson y Grines, de la City University of London, publicado en el New Scientist, el 18 de febrero de 1988, bajo el título: "Lifting scales from our pipes".

A fines del siglo pasado, el Departamento de Energía de Estados Unidos evacuó un reporte de alerta federal referente a la aplicación de tecnologías no químicas para el control de incrustaciones y dureza, y afirma que el control magnético de incrustaciones puede reemplazar a la mayor parte de los equipos ablandadores de agua. Específicamente, ablandadores químicos como la cal, el intercambio iónico y la ósmosis inversa, cuando son usados para controlar la dureza, potencialmente pueden ser reemplazados por tecnología no química de acondicionamiento de agua. Esta incluiría aplicaciones para tratamiento de agua en torres de enfriamiento y para calderas, tanto en sistemas abiertos como cerrados. En dicho reporte se describen diversas tecnologías para tratar agua, entre las que destaca el tratamiento magnético, donde se menciona que gracias al avance de la ciencia de los materiales y a la disponibilidad de elementos magnéticos de alto poder como las aleaciones de tierras raras, se ha hecho más confiable el tratamiento magnético del agua.

\section{PROBLEMA}

El mayor número de problemas cotidianos causados por el agua dura en nuestro país son: formación de sarro en los conductos, corrosión generalizada, aumento del número de145 reparaciones, mayores costos de mantenimiento y funcionamiento, reposición prematura de maquinarias y equipos. Esta situación causa pérdidas de eficiencia, con el consecuente aumento en el uso de energía, lo que genera el impacto de mayores emisiones gaseosas en el ambiente. En resumen, el resultado es 
mayores pérdidas de productividad causadas por paros forzosos de las líneas de producción para descalcificar o reparar la maquinaria, así como también un mayor impacto en el ambiente por uso de productos químicos para tratar el agua. La causa de este problema es la gran aportación de carbonato cálcico en el agua, el cual, a una temperatura dada y a cierta presión, tiende a precipitarse en cristales que forman las incrustaciones calcáreas. Este fenómeno produce dos efectos: calcificación y corrosión.

\subsection{Calcificación}

Cuando el carbonato de calcio se precipita se produce la calcificación, es decir los iones de carbonato de calcio ( $\mathrm{CaCO}$ ) se cristalizan formando incrustaciones calcáreas conocidas como caliche. Por ejemplo, en un agua a $80^{\circ} \mathrm{C}$ los sedimentos se precipitan, formando cristales de calcita y aragonito. La calcita es la forma hexaédrica del carbonato de calcio y se asemeja a adoquines en forma de pequeños bloques que son fáciles de agruparse unos a otros formando estructuras sólidas similares a paredes de una edificación (véase la figura 1). Su apariencia es pétrea y muy difícil de eliminar. Aunque se puede disolver con un lavado ácido, los efluentes vertidos tienen impactos negativos en el ambiente. Por otro lado, se forma el aragonito, cuya figura es esferoidal (tal como se observa en la figura 2). Estos cristales son muy difíciles de agrupar unos con otros por ser redondos y normalmente se encuentran en forma de sedimentos. Su estructura es esponjosa y quebradiza, lo cual facilita su limpieza y eliminación.

Figura 1

Cristales de calcita

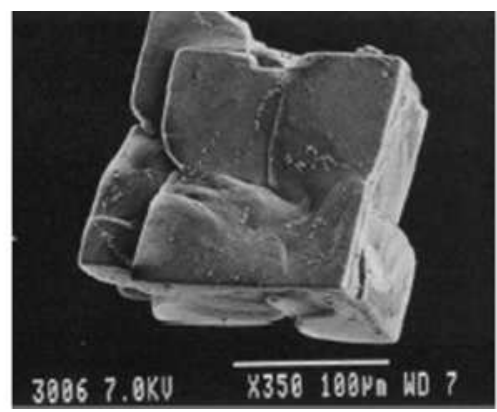

Figura 2

Cristales de aragonito

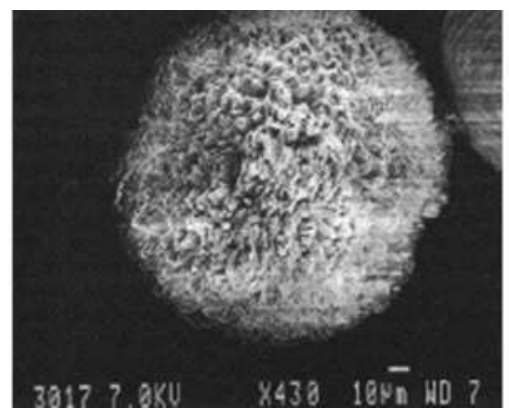

Fuente: $<$ http://www.ucm.es/info/crismine/Sol_PPT/FotosTema4.pdf $>$. 
Luego de un tiempo determinado, que pueden ser algunos años en un caso (cañerías de agua fría de agua de la red doméstica) o de algunos meses en otro (tuberías de uso industrial alimentadas con agua de pozo o tuberías de agua caliente en general), se forman incrustaciones en las líneas por donde circula el agua, reduciendo los diámetros en las tuberías (véase la figura 3) y reduciendo el caudal del flujo de agua, con las consecuentes pérdidas de energía en el proceso productivo (véase la figura 4).

Figura 3

Incrustaciones calcáreas

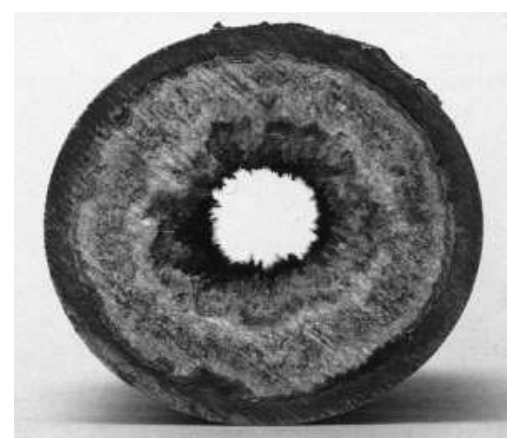

Fuente: Kurita 1999: 10-34.

Figura 4

Cuadro de eficiencia energética vs incrustaciones calcáreas

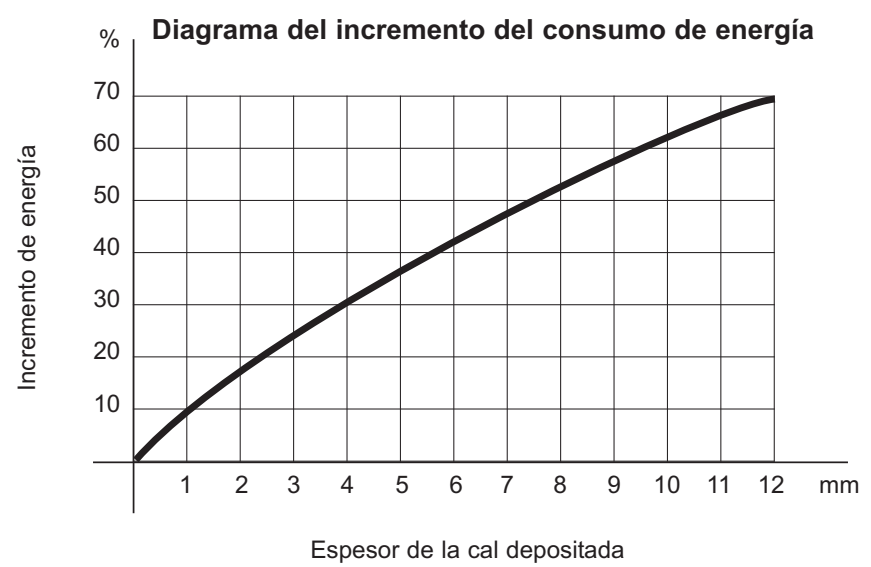

Elaboración propia. 
Por lo tanto, se genera una sobrecarga de energía para conseguir los mismos resultados que con un circuito libre de incrustaciones. En la práctica, el diagrama mostrado significa lo siguiente:

- Obstrucción en las tuberías, lo que implica:

- Disminución de la presión en la red.

- Disminución del caudal en la red.

- Aumento de costos energéticos.

- Aumento de costos de reparaciones y mantenimiento.

- Pérdidas productivas, al existir:

- Resistencias eléctricas incrustadas por cal.

- Mayor tiempo para calefacción o refrigeración.

- Reposición prematura de válvulas, llaves, accesorios y maquinaria en general.

\subsection{Corrosión}

La corrosión es el deterioro de un material metálico como consecuencia de un ataque químico por su entorno. Siempre que la corrosión esté originada por una reacción química, la velocidad a la que tiene lugar dependerá en alguna medida de la temperatura, la salinidad del fluido y las propiedades de los metales en cuestión. La corrosión que habitualmente afecta las tuberías y maquinarias es la corrosión húmeda, un proceso electroquímico que necesita tres factores para desarrollarse espontáneamente: ánodo, cátodo y electrolito (solución acuosa eléctricamente conductora). Si alguno de estos falta la corrosión se detiene. Estos tres elementos constituyen lo que se conoce como pila galvánica o electroquímica. La corrosión se produce en las zonas anódicas, mientras que las catódicas permanecen siempre inalteradas. La corrosión será tanto mayor cuanto mayor sea la conductividad del electrolito. En un agua salina, que tiene una conductividad alta, cualquier proceso corrosivo se verá incrementado en actividad y en velocidad. Un agua dulce será poco conductora, por lo que la corrosión será más lenta y menos activa con relación al primer caso.

Las superficies metálicas habitualmente forman zonas propensas a corroerse (zonas anódicas) debido a varios factores, entre ellos: 
- Deformación del metal.- Cambios en la estructura cristalina del metal causados por el calor, bruscas diferencias térmicas, efectos mecánicos, grietas y fisuras por fatiga, etcétera. La sección deformada tiende a ser corroída (zona anódica) frente a las zonas intactas que no se corroen. La corriente galvánica producida entre ambos se incrementa o disminuye proporcionalmente, de acuerdo con la salinidad del fluido que los rodea.

- Contacto de dos metales diferentes.- Debido a diferencias en sus potenciales de reducción, el metal más activo sufrirá corrosión frente al metal más noble, que se mantiene intacto. La corriente galvánica se incrementa o disminuye de acuerdo con la salinidad del fluido (electrolito).

- Corrosión por efecto Evans (influencia de las incrustaciones).- Un sedimento sobre una superficie metálica origina una zona anódica justamente debajo del depósito, donde la concentración de oxígeno es muy pequeña, en comparación con la periferia. Diferencias en las concentraciones de oxígeno se originan con gran facilidad cuando tienen lugar procesos de incrustación de sales.

Figura 5

Corrosión en tubería

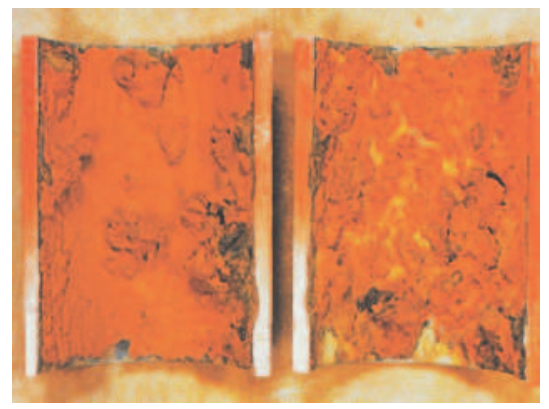

Fuente: Kurita 1999: 2-66.

Las incrustaciones calcáreas o de otras sales minerales en las paredes internas de las tuberías o de la maquinaria producen una diferencia de carga electromagnética entre las moléculas de las sales y los átomos del metal que componen la tubería. Inmediatamente se crea un arco voltaico que genera a su vez el fenómeno de la electrólisis. Esta manifestación electrolítica va escamando el metal de la tubería, y formando lo que comúnmente se conoce como corrosión (véase la figura 5). 
La corrosión acorta la vida útil de las redes por donde circula el agua, así como de la maquinaria y del equipo.

\section{OBJETIVO}

El objetivo del presente trabajo experimental consiste en proponer una alternativa no química para tratar el agua, la que denominaremos desincrustante magnético (DM). Como ya se mencionó, esta tecnología consiste en el uso de campos magnéticos para tratar agua dura con el objeto de revertir el proceso de formación de incrustaciones calcáreas de calcita $(\mathrm{CaCO})$ en la tubería de un intercambiador de calor, en una resistencia de lavadora eléctrica y en una línea de riego por goteo.

El DM consiste en colocar un número determinado de anillos o dispositivos magnéticos que contienen unos imanes permanentes denominados magnetos — de cierta polaridad, orientación e intensidadalrededor de cualquier cañería o manguera de un diámetro de hasta 18 pulgadas, con el objeto de evitar o reducir las incrustaciones de caliche (calcita) en las líneas de agua y en general en cualquier área donde se presenten problemas de incrustaciones.

El DM funciona mediante el uso del poder del magnetismo, el cual es generado por un inductor magnético elaborado de una aleación especial conocida como imán permanente de fuerza industrial y campo magnético enfocado. La polaridad y la intensidad de campo que se suministra al agua son claves para poder apreciar el efecto desincrustante. El DM se instala alrededor de cualquier línea de conducción de agua, en cintas de plástico tipo PVC, HDPE, PP o en tubos de cobre, bronce, aluminio, vidrio, caucho, jebe, acero inoxidable, y en general en cualquier tubería. Para maximizar el efecto del campo magnético en el agua se recomienda reemplazar un tramo de 20 a $40 \mathrm{~cm}$ de la tubería y sustituirlo de preferencia por uno de acero inoxidable de la misma longitud (véase la figura 6). 
Figura 6

Montaje de dispositivos magnéticos en tubería de INOX de $1,5 \mathrm{~mm}$ de espesor

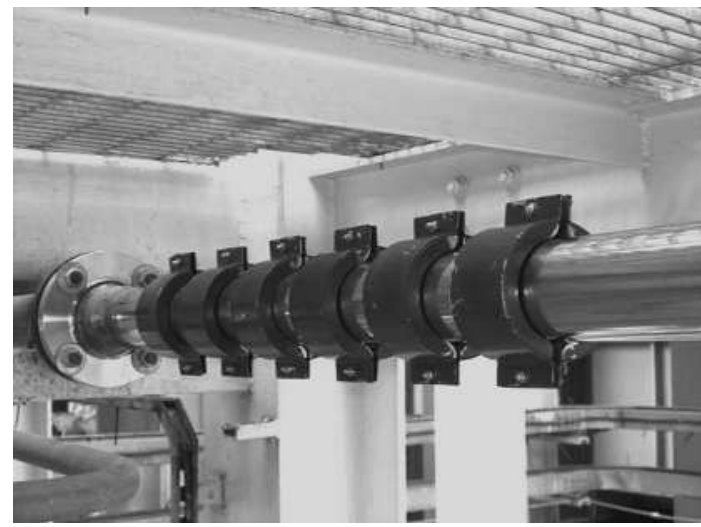

Foto del autor.

\section{METODOLOGÍA}

Se ha evaluado la eficacia de esta tecnología al comparar cada escenario sin $D M$ como con $D M$ para comprobar si hubo alguna variación significativa. Para tal efecto, se realizaron dos pruebas de campo: una de aplicación doméstica y otra de aplicación agrícola, esta última con el objetivo de evaluar el impacto del agua magnetizada en el ambiente. Se instaló el DM en la línea de alimentación de agua de una terma eléctrica y en una línea de riego por goteo de una plantación, respectivamente. En los dos casos se comparó sin DM con los resultados obtenidos con DM.

\section{RESULTADOS}

\subsection{Prueba doméstica: Terma eléctrica}

Para realizar la prueba se seleccionaron dos termas eléctricas de similares características y regímenes de trabajo, con tuberías de alimentación de $\varnothing=1 / 2$ " de fierro galvanizado. En una de ellas se instaló el 
dispositivo DM (figura 7) en la tubería, al cual denominamos con DM. En la otra operó en condiciones normales, denominándola sin $D M$. Se procedió a hacerlas trabajar simultáneamente durante siete años en condiciones similares de operación y con la misma agua de la red del distrito de Surco, en Lima, usando el agua de la red de Sedapal (figura 8).

La resistencia sin DM (figura 8) muestra cierto grado de calcificación, que hubiese ido incrementándose hasta inutilizarla. Sin embargo, en la resistencia de la lavadora que sí trabajó con DM (figura 9) se apre-

Figura 7

Instalación del DM en línea de alimentación de agua fría de térma eléctrica, en la entrada del tanque

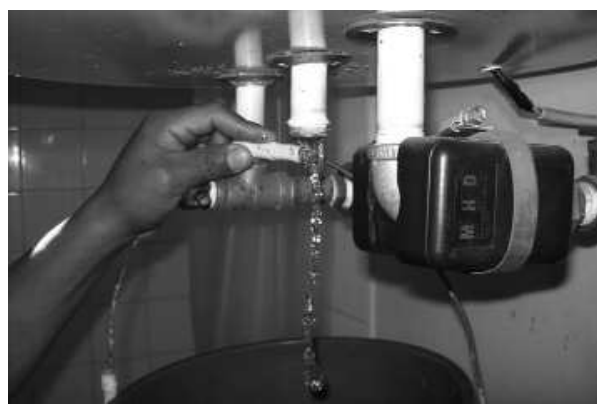

Purga de terma eléctrica de 50lt, luego de 7 años de instalada y con sistema de desincrustante magna hidro dinámica (MHD)

Figura 8

Se muestra la resistencia $\sin D M$

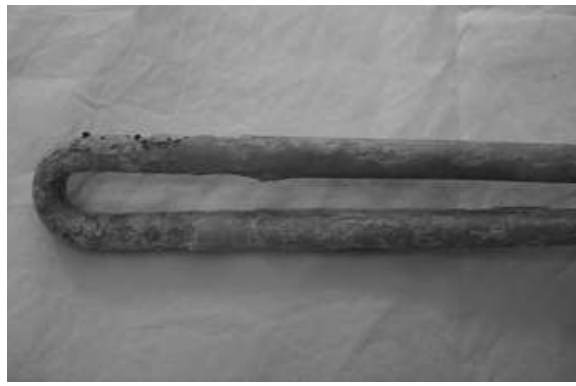

Resistencia de terma sin DM-MHD luego de 7 años (Marca IVYSA, 1.500 Watts)
Figura 9

Se muestra la resistencia con DM

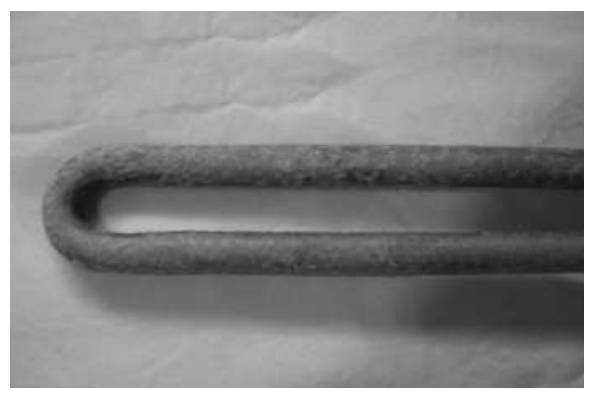

Resistencia de terma con DM-MHD luego de 7 años (Marca IVYSA, 1.500 Watts) 
cia ausencia de calcificación, lo cual garantizará su perfecto funcionamiento por mucho tiempo. Asimismo, se procedió a colectar y pesar los precipitados calcáreos acumulados en el fondo de ambos tanques. Se encontraron 80 gramos en la terma sin DM y 22 gramos en la terma con $\mathrm{DM}$, lo que confirma que con tratamiento magnético del agua se produce una reducción significativa en los depósitos calcáreos.

\subsection{Prueba agrícola: Línea de riego por goteo}

Para realizar la prueba se seleccionaron dos cintas de riego por goteo de idénticas características e igual régimen de trabajo. Se usó una cinta de $\varnothing=17 \mathrm{~mm}$ de plástico en suelo salino e irrigadas con agua de pozo (distrito de Asia, Lima). En una de ellas se instalaron dos dispositivos DM, y la denominamos con DM. En la otra no se instaló DM, y se le denominó sin $D M$. Se procedió a hacerlas irrigar simultáneamente por unas 11 semanas en idénticas condiciones.

Figura 10

Se muestra la planta $\sin D M$ más pequeña

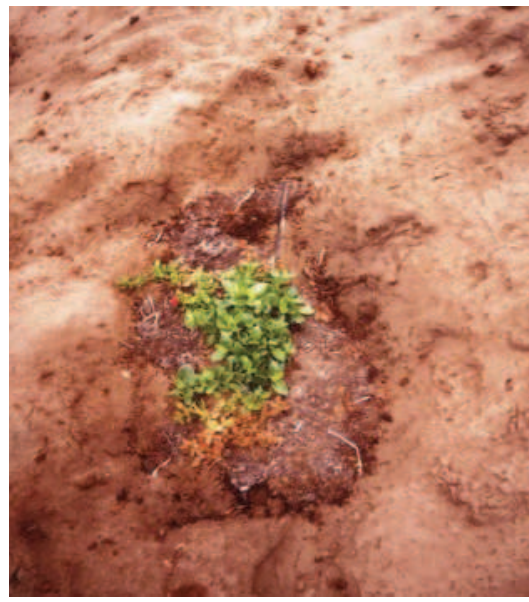

Foto del autor.
Figura 11

Planta con DM, más verde y con hojas más grandes

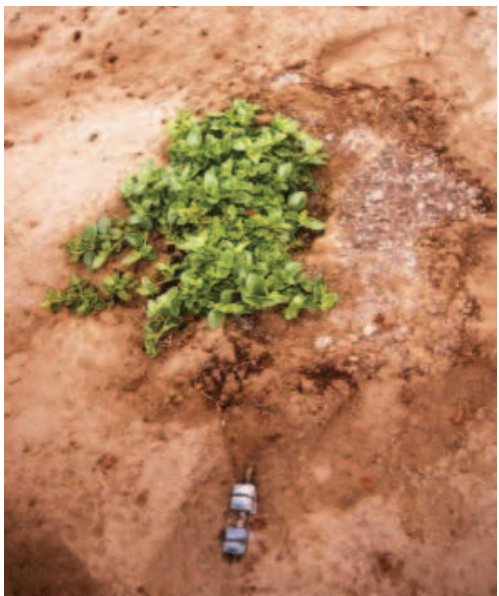

Foto del autor. 
Una vez más se obtuvieron resultados remarcables. Luego de 11 semanas, el sembrío sin DM no se desarrolló debido a que el gotero presentó incrustaciones, taponeándose y haciendo que la planta no se nutra adecuadamente. Se pudo observar también que una parte de esta aparece de una coloración marrón (figura 10), la cual presenta signos de inanición. Sin embargo, la otra planta que sí fue tratada con $\mathrm{DM}$ al cabo de 11 semanas muestra un mayor volumen foliar y mejor coloración, y se observó una mayor floración. Por lo tanto, el agua tratada magnéticamente favorece la vida mejorando la plantación y, lo que es mejor, sin dañar el sustrato o suelo.

\section{DISCUSIÓN}

El enorme problema que plantea el agua dura quedaría resuelto con el uso de DM hasta en un 80\%. El DM reduce la tensión superficial del agua, polarizando su molécula.

El DM se instala en el exterior de las tuberías, por lo tanto no cambia la composición química del agua, sino que varía su estructura física mediante algo tan viejo como nuestro planeta: la energía magnética, una energía natural.

Para que el DM opere adecuadamente deberá ser instalado alrededor de una cañería o conducto de cualquier material, excepto de fierro galvanizado o acerado. En este caso, se deberá reemplazar un tramo del tubo de fierro por uno de INOX 304 (1,5 mm de espesor).

Las características físicoquímicas del agua, su velocidad, presión y temperatura, así como el material y espesor del tubo, son los factores clave para diseñar el arreglo magnético a fin de intensificar el efecto magnetizador en el proceso de cristalización de las incrustaciones.

El mecanismo más probable de activación del proceso de reversión de cristalización sería la Fuerza de Lorentz inducida por dicho tratamiento magnético.

El éxito del uso del DM como método no químico para tratar el agua reside en una amplia gama de aplicaciones y en su eficacia demostrada. Su fácil instalación, que puede ser realizada domésticamente por el mismo usuario sin necesidad de herramientas ni de modificar las conducciones, el no requerir mantenimiento, no consumir ningún tipo de energía, su larguísima duración (hasta 20 años), bajo costo y sobre 
todo por representar un método limpio y no contaminante para tratar el agua son factores que hacen que el DM se pueda usar masivamente, tanto por la industria como por el agro, para mejorar su productividad y elevar la calidad de vida de nuestra sociedad.

El DM puede ser aplicado en el agro, la industria alimentaria, las plantas conserveras, pesqueras, papeleras, mineras y cementeras; asimismo, se puede usar en hospitales, hoteles, restaurantes, colegios, universidades, panaderías, lavanderías, peluquerías, complejos turísticos y deportivos, viveros, invernaderos, sistemas de riego agrícola y ornamental, en los hogares, y en general, en cualquier lugar y circunstancia donde se requiera agua y existan problemas de incrustaciones, ya sea agua de la red (Sedapal y organismos de saneamiento de provincias), o agua de río o de pozo (que alimenta casas de playa, industrias mineras y pesqueras, piscigranjas, tanques, estanques, cisternas, sistemas de riego por aspersión y goteo, etcétera).

El uso del DM tiene las siguientes ventajas: reduce la calcificación hasta en $80 \%$, evita la corrosión en cierta medida, ahorra electricidad, gas licuado de petróleo y gas natural comprimido; produce el efecto de agua blanda, reduce el uso de químicos y agroquímicos hasta en 50\%, reduce el consumo de cloro en las piscinas y los jacuzzi hasta en $50 \%$, alarga la vida útil de las tuberías, llaves, duchas y resistencias de termas, lavadoras y lavavajillas. En aplicaciones agrícolas mejora los índices de germinación, crecimiento, rendimiento y color de las plantas. No degrada el suelo, más bien lo recupera.

El DM no consume energía, no ensucia, no usa químicos, es libre de mantenimiento, y lo más importante, es barato y ya se fabrica en el Perú.

\section{BIBLIOGRAFÍA}

Department of Energy, USA. (Enero de 1998). Federal Technology Alert, DOE/EE-0162, U.S. Washington.

Evans, Ulick R. (1948). An introduction to metallic corrosion. 3. a edición. Londres: Edward Arnold.

J. M. D. Coey, Stephen Cass (2000). Journal of Magnetism and Magnetic Material 209, Dublin: Elsevier. 
Kuivinen, David E. (1975). Comparing corrosion rates of steel corrosion coupons in magnetically treated water and water system utilizing corrosion inhibitors. Cleveland, OH: Lewis Research Center.

López, Alcides et al. (Abril del 2010). "Reporte del análisis químico y cristalográfico de depósitos calcáreos en tanques de termas eléctricas de la empresa". Informe Científico Tecnológico. Lima: Instituto Peruano de Energía Nuclear.

Quinn, C. J.; Molden, Tc. y C. W. Sanderson (Setiembre de 1996). Proceedings iron and steel engineer. Nonchemical approach to hard water scale, corrosion and white rust control. Chicago. 